EPJ Web of Conferences 66, 11003 (2014)

DOI: $10.1051 /$ epjconf/ 20146611003

(C) Owned by the authors, published by EDP Sciences, 2014

\title{
ALPI Setup as the SPES Accelerator of Exotic Beams
}

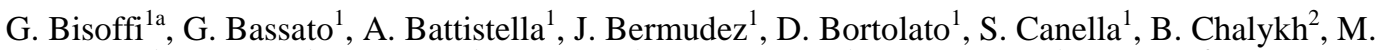
Comunian $^{1}$, A. Facco ${ }^{1}$, E. Fagotti ${ }^{1}$, A. Galatà ${ }^{1}$, M. Giacchini ${ }^{1}$, F. Gramegna ${ }^{1}$, T. Lamy ${ }^{3}$, P. Modanese ${ }^{1}$, A. Palmieri ${ }^{1}$, R. Pengo ${ }^{1}$, A. Pisent ${ }^{1}$, M. Poggi ${ }^{1}$, A. Porcellato ${ }^{1}$, C. Roncolato ${ }^{1}$, D. Scarpa ${ }^{1}$

${ }^{1}$ INFN-Laboratori Nazionali di Legnaro, 35020 Legnaro (Padova), Italy

${ }^{2}$ Institute for Theoretical and Experimental Physics, Moscow, Russian Federation

${ }^{3}$ Laboratoire de Phyisique Subatomique et de Cosmologie, CNRS-IN2P3, Grenoble, France

\begin{abstract}
The SPES (Selective Production of Exotic Species) project for a national exotic beam facility in Legnaro includes pivotal upgrades of the existing superconducting linac ALPI (Acceleratore Lineare Per Ioni), to make it appropriate as the RIB (Radioactive Ion Beam) accelerator. The new injector, consisting of an Electron Cyclotron Resonance (ECR)-type charge breeder and a radiofrequency quadrupole (RFQ), will be described. Upgrade measures in ALPI to improve beam transmission and final energy, and handle low-intensity RIB will be explained, with the aim of increasing transmission to $\mathrm{T}>90 \%, \mathrm{E}_{\mathrm{f}}$ by $\sim 20 \%$, reaching $10 \mathrm{MeV} / \mathrm{u}$ for the reference beam ${ }^{132} \mathrm{Sn}$.
\end{abstract}

\section{Introduction}

The INFN-LNL facility for exotic species, called SPES [1], is in staged construction in Legnaro. Fig. 1 shows the layout of the SPES facility, from the CB and the new injector, to ALPI and the transport lines to the experimental halls.

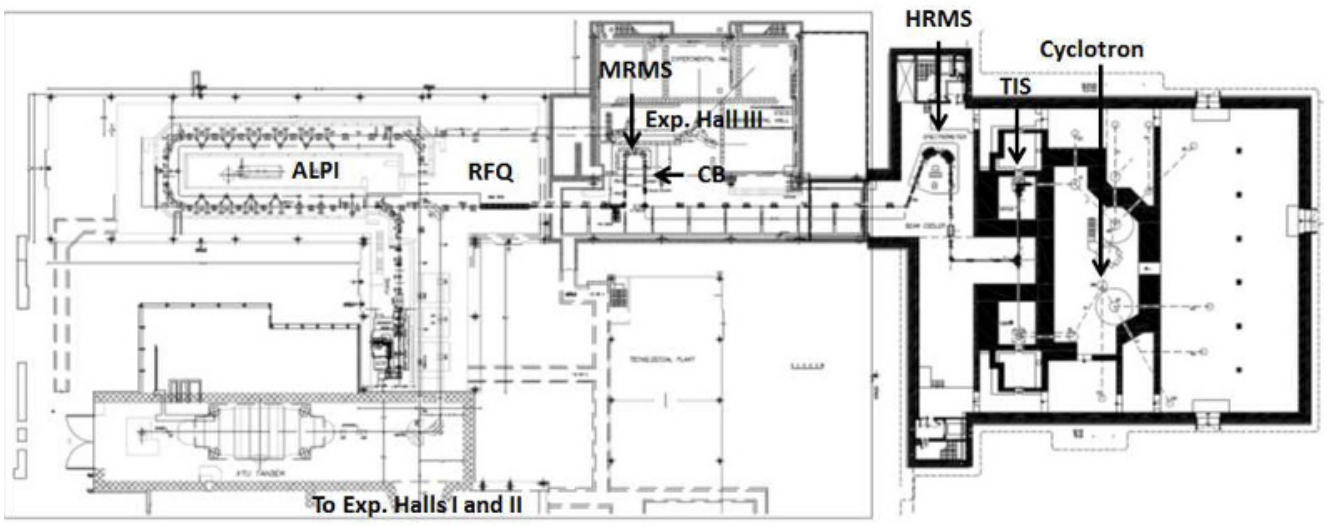

Figure 1. - Layout of the SPES Facility

The SPES accelerator starts with a proton cyclotron $(70 \mathrm{MeV}, 700 \mathrm{uA})$, impinging the beam onto a multislice UCx target, producing exotic species mostly via nuclear fission. The target is connected to

Corresponding author: bisoffi@lnl.infn.it

This is an Open Access article distributed under the terms of the Creative Commons Attribution License 2.0, which permits unrestricted use, distribution, and reproduction in any medium, provided the original work is properly cited. 
an ion source system (either a Surface, Plasma or Laser Ionization Source - SIS, PIS or LIS), from which a $\mathrm{q}=1+$ ion beam is extracted, containing the desired element and related contaminations. The latter are removed first of all through a Wien filter and a high resolution mass separator (HRMS, $\mathrm{M} / \Delta \mathrm{M} \sim 40,000)$ and the purified beam is delivered, through an electrostatic focusing line, to an ECRtype charge breeder (CB). The CB, followed by a Continuous Wave (CW) RFQ, can be seen as the front-end of a novel injector into the superconducting (sc) linac ALPI, which is required to accelerate the RIB species of interest to a final energy $\mathrm{E}_{\mathrm{f}} \sim 10 \mathrm{MeV} / \mathrm{A}$, for experiments in the three experimental halls of LNL. The realization of the new ALPI injector for SPES was funded in 2013, together with some upgrade measures which would make ALPI suitable as an RIB accelerator. This paper will cover both the design of the new injector and such upgrade measures.

\section{The RIB Injector}

After the HRMS charge state 1+ is too low for efficient downstream acceleration: a CB has to be implemented. A 14,5 (18) GHz ECR-type CB was chosen for SPES, to be developed on the basis of the Phoenix booster in operation at LPSC-Grenoble [2,3] and TRIUMF [4]. Typical operational values for the magnetic field are $1.2 \mathrm{~T}$ at injection, $0.42 \mathrm{~T}$ minimum and $0.82 \mathrm{~T}$ at extraction. An $\mathrm{A} / \mathrm{q}$ ratio 6 or lower and a measured emittance $4 * \varepsilon_{\mathrm{rms}} \approx 60 \pi * \mathrm{~mm} * \mathrm{mrad}$, much smaller than the acceptance of the following accelerator complex, nicely fit SPES requirements. Recent results [3] in terms of efficiency and charge breeding time, for typical beam species, are promising. The CB is equipped with a $1+$ source (SIS and PIS types) stable beam line, to provide accurate beam characterization before operating it with RIB's; this line has a $\mathrm{M} / \Delta \mathrm{M} \sim 150$ mass resolving power.

Due to the contaminations introduced by the $\mathrm{CB}$ itself, a $\Delta \mathrm{M} / \mathrm{M} \sim 1 / 1000$ separation is required downstream, to well separate the species of interest. With $\varepsilon_{\mathrm{RMS}, \text { norm }} \sim 0.1 \mathrm{~mm}$ mrad after the $\mathrm{CB}$, the beam size is quite large to be handled at $40 \mathrm{keV}$, i.e. with a $\mathrm{B} \rho=0.075 \mathrm{~T} * \mathrm{~m}$. The preferred solution [5] (fig.2, left side) is to locate a medium resolution mass spectrometer (MRMS) on a $120 \mathrm{kV}$ platform, thus increasing $\mathrm{B} \rho$ to $0.15 \mathrm{~T}^{*} \mathrm{~m}$. With $\rho=750 \mathrm{~mm}$, the dipole field $\mathrm{B}_{\text {dip }}$ is $\sim 0.2 \mathrm{~T}$, a reasonably high value for a not too demanding magnet. Optics elements of the spectrometer are electrostatic lenses and a multipole, so as to compensate for emittance increase. In a first order calculation, beam separation is about 1/2500; macroparticles transport, including non-linear effects and multipole emittance compensation, is shown in fig. 2 (right side) where three beams, separated by $\Delta \mathrm{M} / \mathrm{M} \sim 1 / 1000$ in mass, are split in space at the end of the spectrometer by $\sim 8 \mathrm{~cm}$.
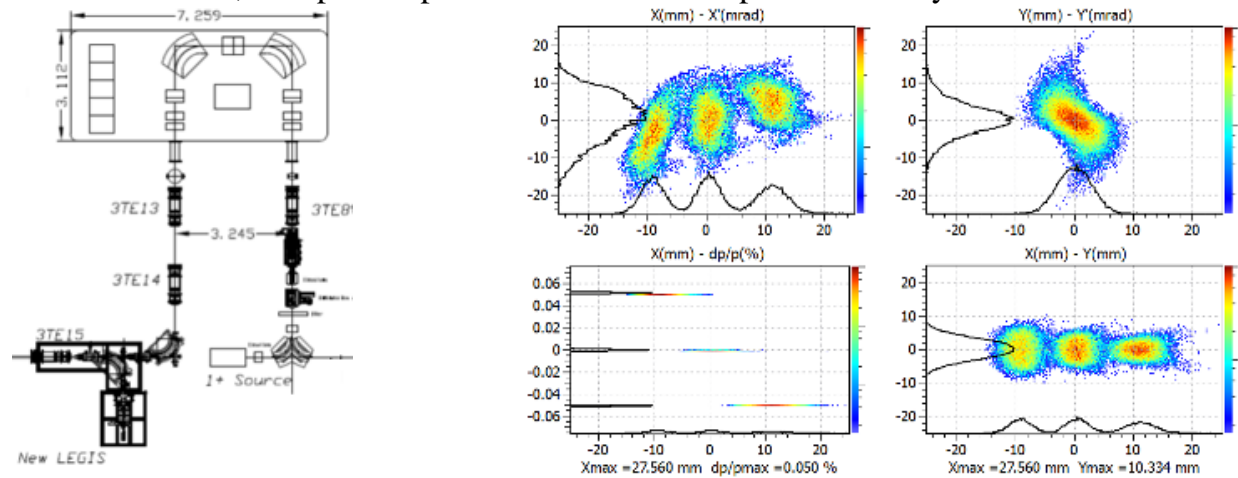

Figure 2. - Layout of the MRMS after the CB (left); macroparticle simulation, showing 1/1000 mass separated beams at the end of the spectrometer (right).

After the MRMS a Medium Energy Beam Transport (MEBT) line (fig.1) matches the beam to the injection acceptance of a CW normal conducting RFQ, which imparts to the RN beam the energy required by the velocity profile $\left(\beta_{\mathrm{opt}}\right)$ at the entrance of ALPI. 
The RFQ [6] will operate $\mathrm{CW}$ at $80 \mathrm{MHz}$ (same frequency as the lowest energy ALPI superconducting cavities). The injection energy, set at $5.7 \mathrm{keV} / \mathrm{u}$, is a compromise between the aim of reducing the ion energy to simplify Low Energy Beam Transport (LEBT) line and RFQ bunching section design on one side, and the need to increase the injection energy to increase the beam rigidity in the spectrometer and to reduce space charge effects on the other. A rather high extraction energy (727 keV/u) optimizes the beam dynamics of the linac. The RFQ design is such that a beam with $\varepsilon_{\text {RMS,norm }} \sim 0.15 \mathrm{mmmrad}$, with T $\sim 90 \%$, calculated on a $6 \sigma$ Gaussian distribution, may be transported.

The SPES RFQ is a four-vane structure, divided in 7 modules of approximately equal length. The cylindrical tank (800 mm diameter) is in Cu-plated annealed stainless steel (SS). Electrodes are built in $\mathrm{Cu}$ and SS, where brazing under vacuum is used for the cooling channels and the interface reference surfaces between electrode and tank. The electrode modulation is milled to its final value after brazing, and - as the last construction stage - electrodes are positioned, aligned and bolted to their final position in the tank. Fig. 3 shows the RFQ geometry (left) and its main RF parameters (right).

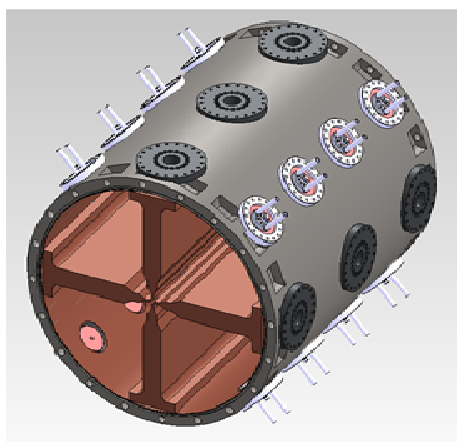

\begin{tabular}{|l|l|l|}
\hline $\begin{array}{l}\text { Shunt Inpedance } \\
\text { (SF) }\end{array}$ & $\begin{array}{l}538- \\
552\end{array}$ & $\mathrm{k} \Omega \cdot \mathrm{m}$ \\
\hline $\mathrm{Q} 0$ (SF) & 20000 & \\
\hline $\begin{array}{l}\text { Copper power } \\
\text { (SF) }\end{array}$ & 73 & $\mathrm{~kW}$ \\
\hline Stored Energy & 3.0 & $\mathrm{~J}$ \\
\hline Max H field (2D) & 1633 & $\mathrm{~A} / \mathrm{m}$ \\
\hline $\begin{array}{l}\text { Max Power } \\
\text { Density (2D) }\end{array}$ & 0.31 & $\mathrm{~W} / \mathrm{cm}^{2}$ \\
\hline Total Power & 113 & $\mathrm{~kW}$ \\
\hline
\end{tabular}

Figure 3. - Mechanical layout of a 1 meter long sample RFQ tank module (left); main RF parameters (right).

\section{RIB-Upgrade of the superconducting linac ALPI}

For its use as RIB accelerator, the sc linac ALPI is undergoing some upgrade actions aiming at: dealing with low-I beams (better beam transmission and provision of low-I beam diagnostics); increasing the final energy, up to $~ 10 \mathrm{MeV} / \mathrm{A}$ for the RIB species of reference ${ }^{132} \mathrm{Sn}^{18+}$; improving the accelerator reliability (more efficient cryogenics, new vacuum system, updated and integrated control system). The present configuration of ALPI fits the requirements for SPES post acceleration too. However, an upgrade of its performances both in overall transmission and final energy is required [7]. First of all, higher energy from the new RFQ injector, as explained above, will allow to better match beam features to the medium- $\beta$ cryostats, where RF defocusing is too strong and the beam could get easily lost onto the cavity beam ports. Secondly, it has to be noted that, in the last decade, the average cavity accelerating field has been increased by more than a factor of 2 with respect to the original design value [8], while maximum strength of the focusing lenses, on the other hand, remained unchanged $(20 \mathrm{~T} / \mathrm{m})$ : therefore it is hard to design a longitudinal beam dynamics which would not impact on the transverse plane adversely. To fully exploit the available acceleration gradient, improvements are required on ALPI: the 8 QWRs presently installed in the injector PIAVE (Positive Ion Accelerator for low-VElocity Ions) will become the first ALPI cryostats, thus reducing beam losses in the linac to below $\sim 20 \%$, while increasing $\mathrm{E}_{\mathrm{f}}$; two cryostats will be added at the end of ALPI high-E branch, increasing $\mathrm{E}_{\mathrm{f}}\left({ }^{132} \mathrm{Sn}^{18+}\right)>10 \mathrm{MeV} / \mathrm{A}$ [7].

In 2012, an alignment campaign of heavy ion accelerator facilities at LNL started, with the purpose of improving beam transmission and making the accelerator setup for experiment swifter: the procedure included deliberate vertical displacement of ALPI QW resonators from the beam axis, to compensate the QWR vertical steering effect caused by the anisotropy of the QWR geometry [9].

R\&D activity is being carried out for low-I diagnostic tools to be added along ALPI and its injection line: ALPI upgrade requires that those diagnostic boxes, equipped at present with stable 
beam diagnostics, be added tools suited for the extremely low I values of RIB beams $\left(10^{5} \div 10^{8} \mathrm{p} / \mathrm{s}\right)$ : for this purpose beam position and profile monitors, based essentially on microchannel plates (MCP) [10], were already tested with success for a $10 \mathrm{fA}{ }^{40} \mathrm{Ca}$ stable beam.

In two locations along the beam line, after the HRMS and the MRMS, two diagnostic boxes for identification of the RIB species («tape stations») will be implemented, consisting in a moving mylar tape system and a $\gamma$-ray counting chamber, with Ge detectors and plastic detectors to detect positron decays. Fig. 4 shows the tape system setup (left) and the MCP system being developed (right).
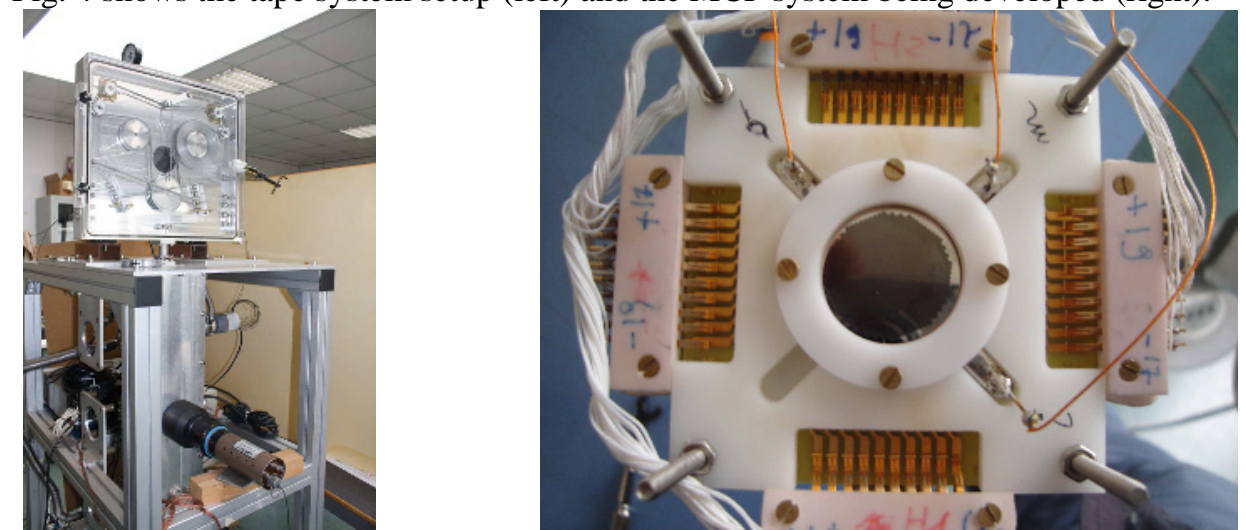

Figure 4. - Being developed RB tape system (left); microchannel plate interceptive RIB diagnostics (right).

Even long-used systems such as ALPI cryogenics and vacuum plants need being consolidated or upgraded in the SPES framework: a $3^{\text {rd }}$ turbine was added to the Air Liquide cryogenics cold box, resulting in an increase of the plant refrigeration capability by more than $50 \%(360 \mathrm{~W})$; as for the vacuum system, the albeit small possible contamination of the vacuum exhaust line (due to losses of RIB beam and isotopes with long $\tau_{\text {decay }}$ ) might dictate ad-hoc conditions on the vacuum system setup; maintenance, waste storage and disposal of the pumps shall have to comply with special procedures and equipment choices. An experimental programme has been started, to study the possible options.

Finally, the LNL accelerator complex is undergoing a major upgrade of its control systems in order to integrate seamlessly with the operation of the overall SPES. The new control system (CS) will use modern network technologies to distribute its action on the campus area and will rely on networked services to fulfil its goals. The control software is based on EPICS [11]. As part of the upgrading process, beam diagnostics software and magnet PS control system are under development. Due to obsolescence of some key components, LLRF (Low Level Radio Frequency) electronics of sc cavities is also being redesigned: a direct sampling digital RF controller is being prototyped and applied to 80 and $160 \mathrm{MHz}$ cavities.

\section{References}

1. G. Prete, these proceedings.

2. T. Lamy et al., Rev. Sci. Instrum. 83, 02 A913 (2012); http://dx.doi.org/10.1063/1.3673629

3. T. Lamy et al, Proc. of ECRIS-2012, Sydney (Australia), to be published.

4. F. Ames et al., Rev. Sci. Instrum. 81, 02A903 (2010).

5. M. Comunian, et al., Proc. of LINAC2012, Tel-Aviv, Israel, 546 (2012)

6. M. Comunian, et al., Proc. of LINAC2012, Tel-Aviv, Israel, 951 (2012)

7. G. Bisoffi et al., Proceedings of HIAT12, Chicago, IL, USA, 106 (2012)

8. G. Bisoffi et al., Proc. SRF2013, Beijing, China, 55 (2007)

9. A. Facco and V. Zviagintsev, Phys. Rev. ST Accel.\&Beams 14, 070101 (2011)

10. J. L. Wiza, Nuclear Instruments and Method 162, 587 (1979)

11. Dalesio L. R. et al., Proc. of the Int. Conf. on Accelerators and Large Experimental Physics Control Systems, Tsukuba, Japan, 278 (1991). 